$\xi=-1$

\title{
Modelling of Horizontal Flexible Plate Structure Using Artificial Bee Colony Algorithm
}

\author{
Muhamad Sukri Hadi ${ }^{1 *}$, Intan Zaurah Mat Darus² \\ ${ }^{1}$ Department of Dynamics, Control and Systems Engineering, Faculty of Mechanical Engineering, Universiti Teknologi MARA, 40450 \\ Shah Alam, Selangor, Malaysia. \\ ${ }^{2}$ Department of Applied Mechanics and Design, School of Mechanical Engineering, Faculty of Engineering, Universiti Teknologi Malay- \\ sia, 81310 Johor Bharu, Malaysia. \\ *Corresponding author E-mail:msukrihadi@uitm.edu.my
}

\begin{abstract}
This paper presents the performance of system identification for modeling the horizontal flexible plate system using artificial bee colony and recursive least square algorithms. Initially, the experimental rig of flexible plate was designed and fabricated with all edges clamped boundary condition at the horizontal position. Then, the instrumentation and data acquisition systems were integrated into the rig for acquiring the input-output vibration experimentally. The collected data in the experiment will be used later for modeling the dynamic system of horizontal flexible plate system using system identification. The effectiveness of the developed model will be validated using mean squared error, one step ahead prediction, correlation tests and pole zero diagram stability. The estimated of the developed models were found are acceptable and possible to be used as a platform of controller development for vibration suppression of the undesirable vibration in the flexible plate structure. It was found that the artificial bee colony algorithm has performed better in this study by achieving the lowest mean squared error, good correlation test and high stability in the pole zero diagram.
\end{abstract}

Keywords: Active vibration control, Artificial bee colony, Evolutionary swarm algorithm, Flexible structures, System identification.

\section{Introduction}

The usage of flexible plate structure has been growing faster and widely used in the various engineering applications such as mechanical, automotive, aerospace and marine. The advantages possess by the flexible structure such as light, reliable and efficient have attracted the industries to shift for employing the flexible structure rather than using a rigid structure. Beyond, of the advantages, it is also has its downside in the engineering applications which are the light weight characteristic of flexible structure can be easily influenced by the external vibration $[1,2]$.

The undesirable vibration occurs on the flexible structure has given a major problem to the industries including instability, fatigue, bending and low performance. Therefore, controlling the undesirable vibration on the flexible plate is compulsory. Since then, a huge attention has been given by the researchers due to solve the vibration problem on the flexible plate structure [3].

Flexible structure is known as a light and thin structure when subjected to the disturbance force, it would demonstrates an intrinsic property of vibration which can lead to the failure of the structure. The element of flexible structure for flexible structure such as shells, frames, been and plate. However, the most commonly used in the industrialized world is a flexible plate structures $[4,5]$.

Many methods have been proposed by the researchers in order to suppress the vibration by considering several control strategy. But, a key to design the best controller to suppress the vibration in the system is finding an appropriate model of the flexible structure system. One of the robust method to find an accurate model of dynamic system is using system identification technique [6]. An observed input-output vibration data need to be acquired in order to characterize the dynamic of the system using system identification technique. Although, the method has been widely used by many researchers, but, it is still consists an open area to conduct the research due to complex dynamic of the flexible structures [7]. The main purpose of this study is to develop a modeling of horizontal flexible plate system using system identification technique via artificial bee colony and recursive least square algorithms based on input-output vibration data acquired experimentally. Initially, the experimental rig will be designed and fabricated with all edges boundary condition at the horizontal position. Then, the instrumentation and data acquisition systems will be integrated into the rig for vibration data collection purpose. The data acquired in the experiment will be used for modeling the dynamic system of flexible structure using artificial bee colony and recursive least square algorithms. The effectiveness of the developed models will be verified using mean squared error, one step ahead predication, correlation tests and pole zero diagram stability.

\section{Experimental setup}

The purpose of conducting this experimental setup is to collect the input-output vibration data of the flexible plate experimentally. Firstly, the experimental rig to be used in this research is designed and fabricated. The flexible structures used in the experiment are square, thin and flat aluminium plate with the dimensions $0.7 \mathrm{~m}$ $0.7 \mathrm{~m} 0.001 \mathrm{~m}$ is considered. The flexible plate was placed in the horizontal position to allow it vibrate vertically. The rig is designed with all the edges are clamped. The full specifications of the flexible plate used in this research are listed in Table 1. 
The magnetic shaker and a circular shape permanent magnet was applied at the excitation point on the experimental rig due to generate an actuation force during conducting the experiment. A circular shape permanent magnet was located at distance $1 \mathrm{~cm}$ parallel to the magnetic shaker at the excitation point. Then, the magnetic shaker was connected to the power amplifier (type 2706) and function generator (GFG-8250A) to create a sinusoidal actuation force for exciting the plate. The integration of the instrumentation used in the experiment was shown in Figure 1.

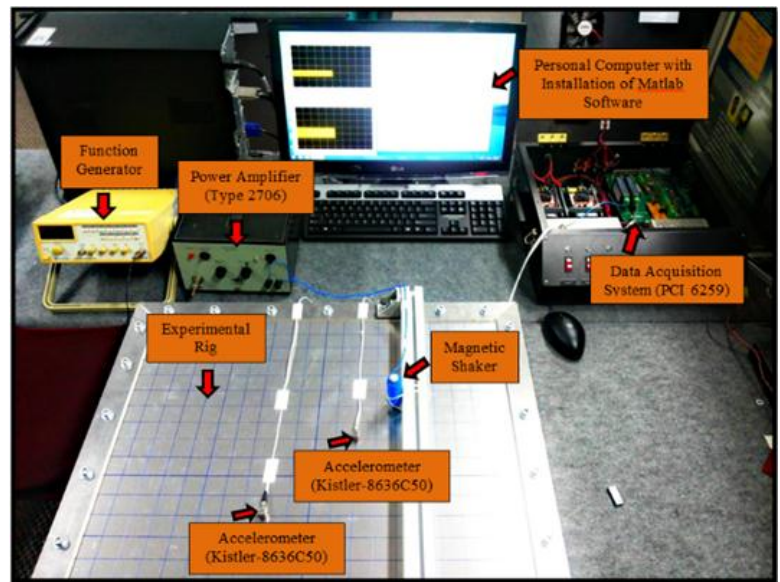

Fig. 1: Experimental setup used in this study.

Two pieces of piezo-beam type accelerometer (Kistler-863650C) were used for sensing the acceleration signal to represent the vibration of the structure. The accelerometers were attached at two different positions which are at the observation and detection points as shown in Figure 2. The piezo-beam type accelerometers were directly connected to the data acquisition system (PCI 6259) which is mounted inside the computer on a PCI-bus connected with SCC-68 through shielded cable. A personal computer equipped with Intel ${ }^{\circledR}$ Core ${ }^{\mathrm{TM}}$ i7-4770K Processor, 4.00 GB RAM and MATLAB software was used to analyze the required signal obtained in the experiment.

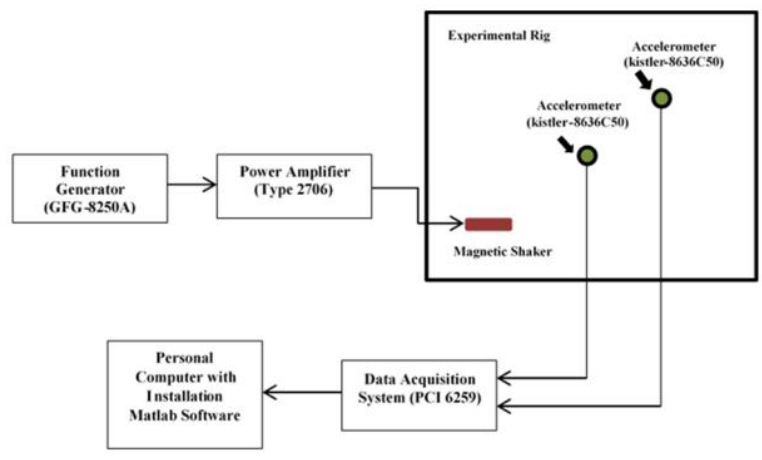

Fig. 2: The layout of experimental setup used in this study.

Table 1: The Specifications of Flexible Plate

\begin{tabular}{cc} 
Table 1: The Specifications of Flexible Plate \\
\hline Parameter & Value \\
\hline Length, $L$ & $0.7 \mathrm{~m}$ \\
Width, $w$ & $0.7 \mathrm{~m}$ \\
Number of sections & $20 \times 20$ \\
Thickness, $T$ & $0.001 \mathrm{~m}$ \\
Moment of Inertia, $I$ & $5.1924 \times 10^{-11} \mathrm{kgm}^{2}$ \\
Mass density per area, $\rho$ & $2.17 \times 10^{3} \mathrm{~kg} / \mathrm{m}^{3}$ \\
Young's modulus, E & $7.11 \times 10^{10} \mathrm{~N} / \mathrm{m}^{2}$ \\
Poison ratio & 0.3 \\
\hline
\end{tabular}

\section{System identification}

In this study, the parametric modelling was conducted using conventional algorithm known as recursive least square (RLS) and intelligent swarm algorithm known as artificial bee colony (ABC) with linear auto regressive with exogenous input (ARX) model structure. RLS is a conventional algorithm developed by Gauss in 1821 that can be used to solve the engineering problem by introducing adaptive fitlters. Details of RLS algorithm can be discovered in this article [8]. Meanwhile, ABC algorithm was introduced by Karaboga to solve the numerical optimization problem based on foraging behavior of bee colony. Details of $\mathrm{ABC}$ algorithm can be found in this paper [9].

In this research, the input-output vibration dataset was acquired through experimentally and utilised in the modelling development. There were 5000 input-output vibration datasets were collected in the experiment and divided into two parts. The first 2500 datasets were used to train the model, whereas, another 2500 datasets were used for testing the performance of the developed model. The developed model was verified in this study due to validate the effectiveness of the developed model. The vibration of the horizontal flexible plate structure in frequency domain was obtained using fast Fourier transform and the first three natural frequencies of the structure were acquired from the frequency response.

\section{Result and discussion}

Heuristic method was used to identify the most suitable model order since there is no prior knowledge on actual model order for horizontal flexible plate structure. For identification using ABC algorithm, the model was tuned properly by varying the orders, number of colony size, number of food sources and number of generations. The best model order was found to be model 8 which consists 16 of number of parameters. The satisfactory results were achieved in $\mathrm{ABC}$ modelling using the following set of parameters as described in Table 2.

Table 2: The set of Parameters Used in ABC Algorithm

\begin{tabular}{cc}
\hline Parameter & Value \\
\hline Number of colony size & 10000 \\
Number of food sources & 5000 \\
Number of generation & 150 \\
Limit range search boundary & 0.55 \\
Model order & 8 \\
Number of parameter & 16 \\
\hline
\end{tabular}

The best mean squared error achieved by $\mathrm{ABC}$ modelling are $1.7459 \times 10^{-5}$ and $4.8337 \times 10^{-6}$ for training and testing data, respectively. The mean square error versus number of generations has been plotted as in Figure 3. Both actual and predicted output of the flexible plate system in time and frequency domains are plotted in the Figures 4 and 5, respectively. Based on Figures 4 and 5 were proved that the model developed is able to imitate the measured output very well. Figure 6 shows the error between actual and estimated output achieved using ABC modelling.

The pole-zero diagram and correlation tests are depicted as shown in Figures 7 and 8, respectively. According to Pole-zero diagram, it is noticed that the model was stable which all the poles of the transfer function in the unit circle. The correlation tests were carried out to determine the effectiveness of the developed system. $\mathrm{ABC}$ modelling was indicated that the model is not biased when the results were found within $95 \%$ of confidence level. The discrete transfer function obtained using $\mathrm{ABC}$ modelling are described in Table 3. 


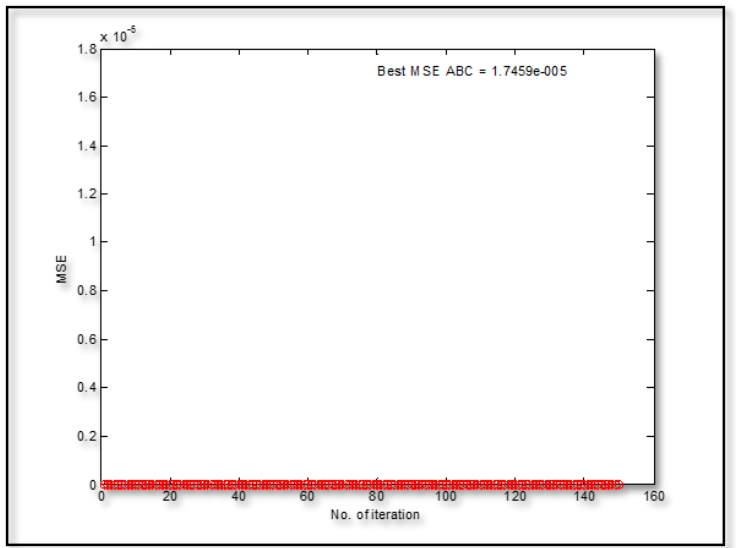

Fig. 3: Artificial bee colony algorithm convergence.

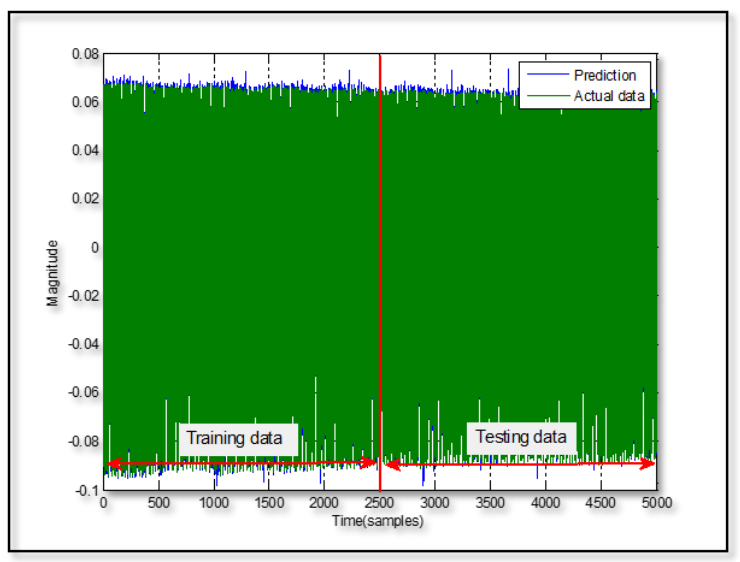

Fig. 4: Actual and estimated outputs of the system in time domain using ABC algorithm.

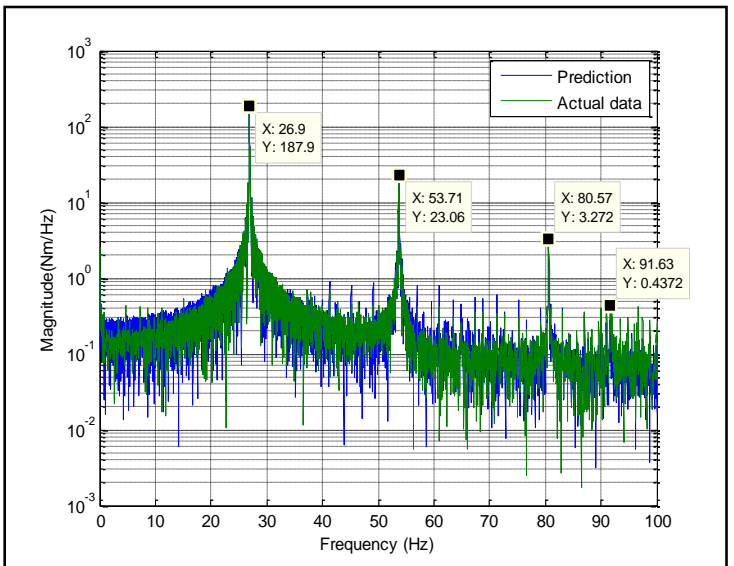

Fig. 5: Actual and estimated outputs of the system in frequency domain using $\mathrm{ABC}$ algorithm.

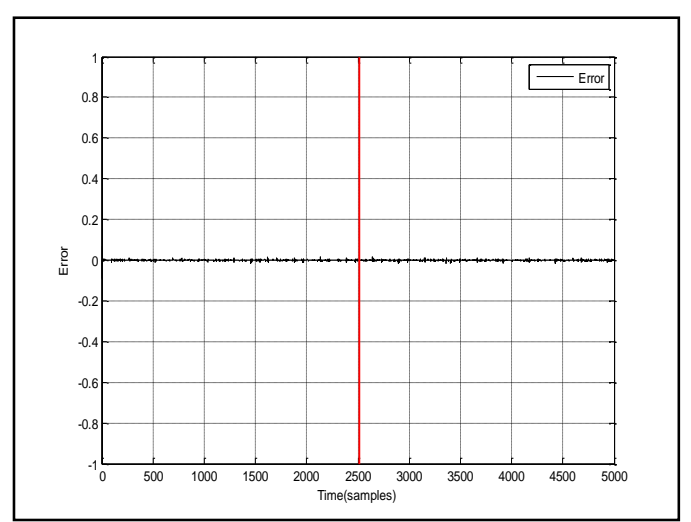

Fig. 6: Error between actual and estimated outputs of the system using $\mathrm{ABC}$ algorithm.

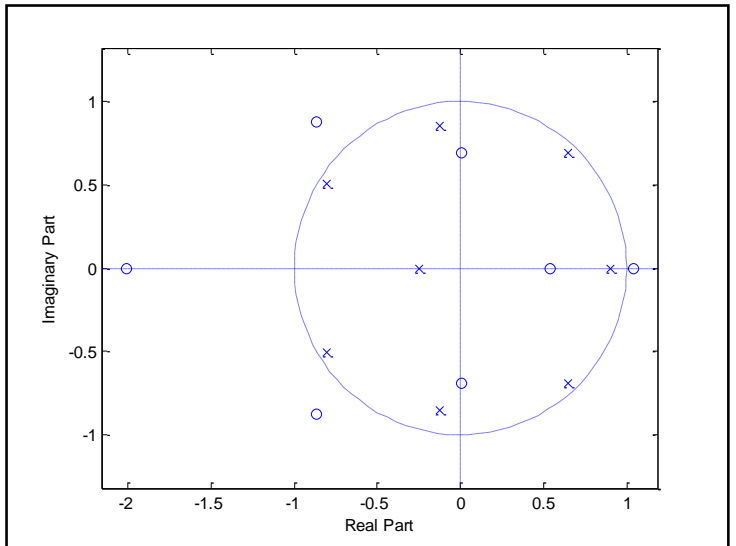

Fig. 7: Pole zero diagram of the system using $\mathrm{ABC}$ algorithm.

Table 3: Transfer function obtained using ABC algorithm

\begin{tabular}{c|c} 
Parameter & Value \\
\hline
\end{tabular}

$\begin{array}{cc}\mathrm{a}_{1}=0.0920 & \mathrm{~b}_{1}=0.2567 \\ \mathrm{a}_{2}=-0.0554 & \mathrm{~b}_{2}=0.5500 \\ \mathrm{a}_{3}=-0.0340 & \mathrm{~b}_{3}=0.0258 \\ \mathrm{a}_{4}=0.2574 & \mathrm{~b}_{4}=-0.4356 \\ \mathrm{a}_{5}=-0.1269 & \mathrm{~b}_{5}=-0.5500 \\ \mathrm{a}_{6}=0.1958 & \mathrm{~b}_{6}=0.1123 \\ \mathrm{a}_{7}=-0.4891 & \mathrm{~b}_{7}=-0.2564 \\ \mathrm{a}_{8}=-0.1348 & \mathrm{~b}_{8}=0.2107\end{array}$

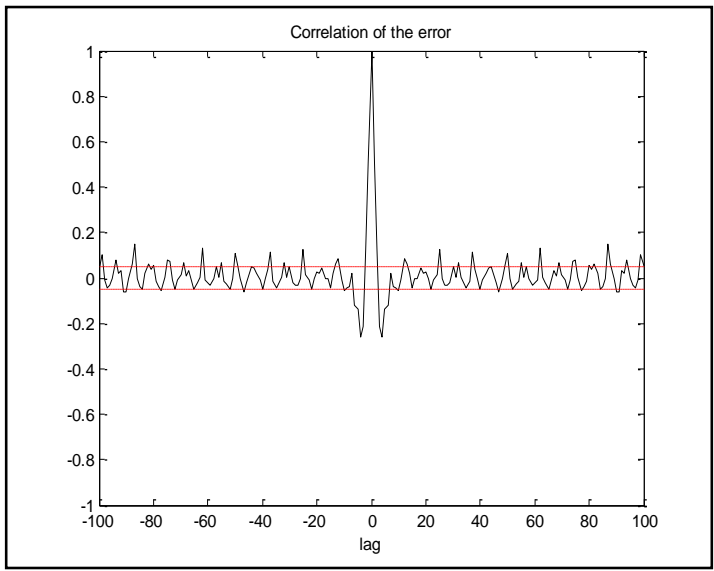

(a)

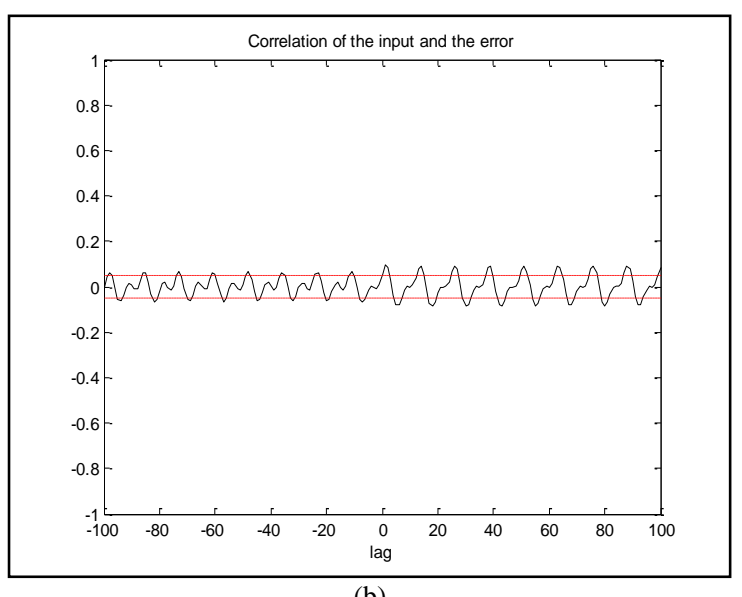

(b)

Fig. 8: Correlation tests for $\mathrm{ABC}$ algorithm: (a) Auto correlation, (b) Cross correlation.

The performance of $\mathrm{ABC}$ modeling was then compared to the recursive least square (RLS) modeling in order to validate the developed model in this study. Similar to ABC, identification using RLS was also conducted using heuristic method to achieve the best model that represent the characteristic of the system. In RLS modeling, the model was tuned properly by varying the or- 
ders and forgetting factor until the satisfactory result was achieved.

The best model order was found to be $10^{\text {th }}$ order with forgetting factor, $\lambda=0.4$. The best and lowest mean squared error for RLS identification are $2.8 \times 10^{-3}$ and $5.4768 \times 10^{-4}$ for training and testing data, respectively. Both actual and predicted outputs of the flexible plate system in time and frequency domains are plotted in the Figs. 9 and 10, respectively. Figures 9 and 10 were showed that the model developed is able to imitate the measured output very well. Figure 11 shows the error between actual and estimated output achieved using RLS modeling.

The pole-zero diagram and correlation tests are depicted as shown in Figures 12 and 13, respectively. As seen in the Pole-zero diagram, noticed that the model is stable which all the poles of the transfer function in the unit circle. The correlation tests were carried out to determine the effectiveness of the developed system. Two correlation tests were used to validate the model which are auto correlation and cross correlation. Through correlation tests the model is indicated to be biased when the results were exceeding the $95 \%$ confidence level. The transfer function obtained using RLS modeling is described as in Table 4.

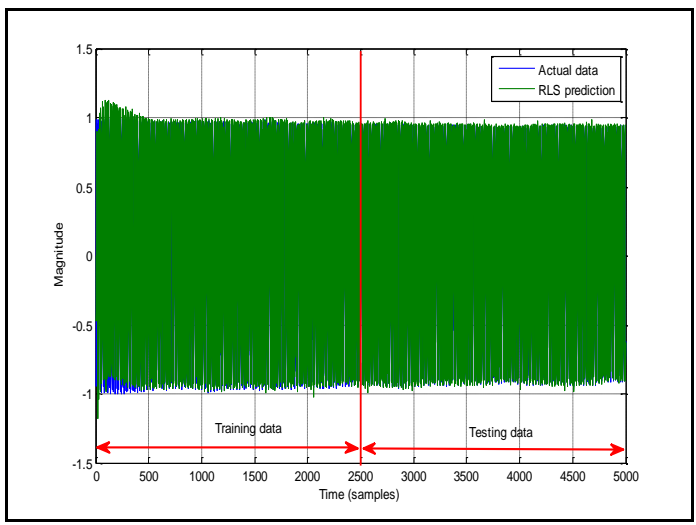

Fig. 9: Actual and estimated outputs of the system in time domain using RLS algorithm

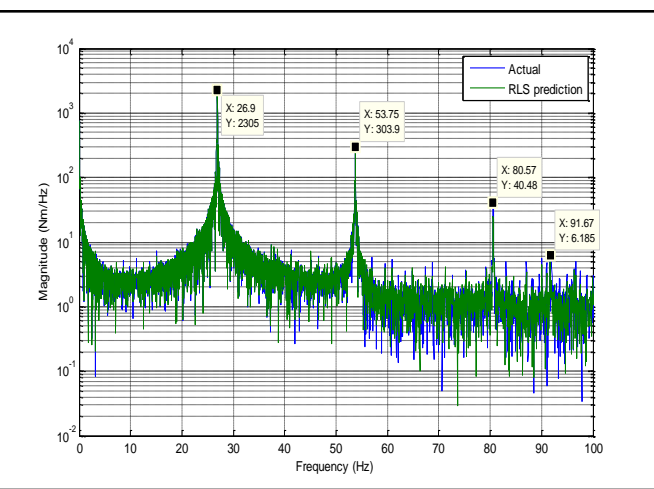

Fig. 10: Actual and estimated outputs of the system in frequency domain using RLS algorithm.

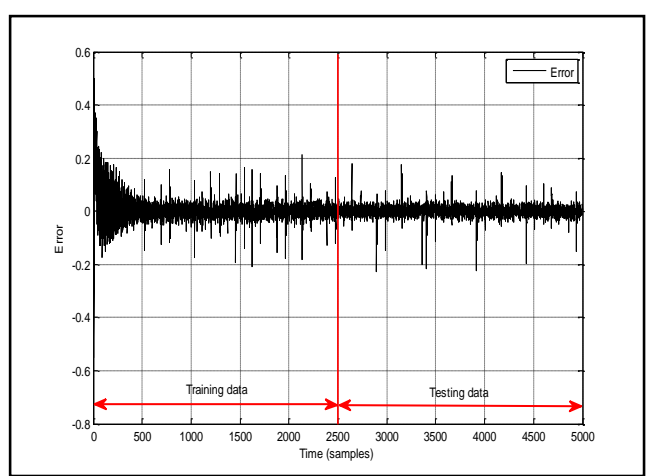

Fig. 11: Error between actual and estimated outputs of the system using RLS algorithm.

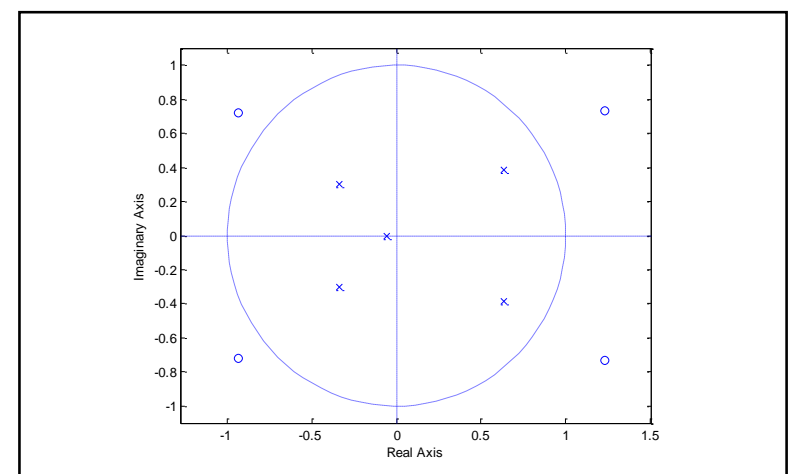

Fig. 12: Pole zero diagram of the system using RLS algorithm.

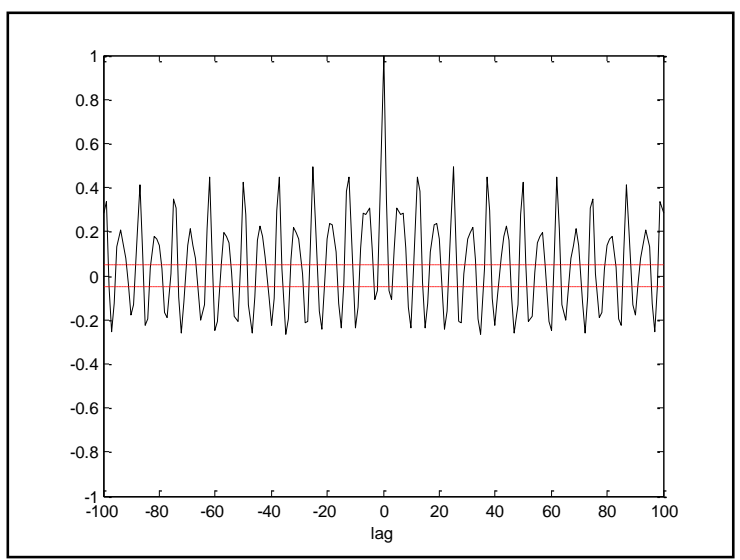

(a)

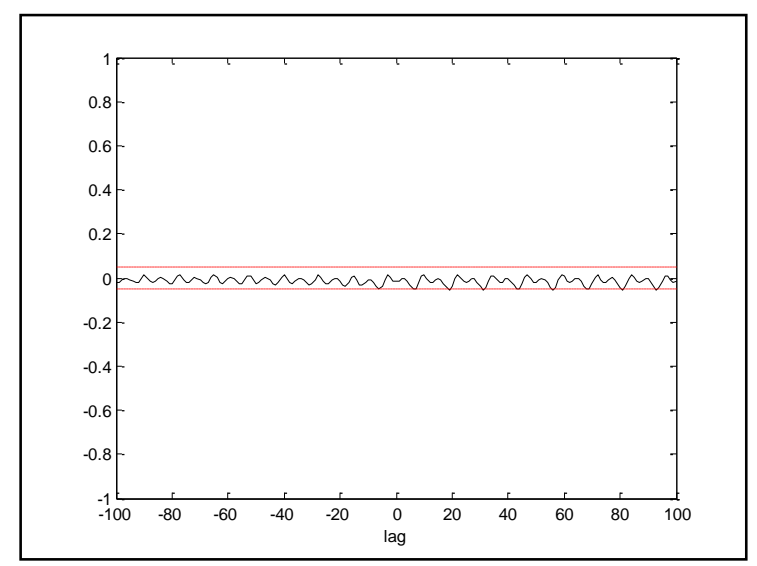

(b)

Fig. 13: Correlation tests for RLS algorithm: (a) Auto correlation, (b) Cross correlation.

Table 4: Transfer function obtained using RLS algorithm

\begin{tabular}{cc}
\hline Parameter & Value \\
\hline $\mathrm{a}_{1}=-0.5326$ & $\mathrm{~b}_{1}=-0.2057$ \\
$\mathrm{a}_{2}=-0.1379$ & $\mathrm{~b}_{2}=0.121$ \\
$\mathrm{a}_{3}=0.1041$ & $\mathrm{~b}_{3}=0.2409$ \\
$\mathrm{a}_{4}=0.1192$ & $\mathrm{~b}_{4}=-0.08509$ \\
$\mathrm{a}_{5}=0.006274$ & $\mathrm{~b}_{5}=-0.5895$ \\
\hline
\end{tabular}

\section{Conclusion}

This paper presented and discussed the dynamic characterization of horizontal flexible plate structure using artificial bee colony and recursive least square algorithms. The models were evaluated and the performances of the models have been assessed. The estimated model using ABC and RLS algorithms have been obtained, verified, acceptable, and possible to be used further for the controller development in order to suppress the unwanted vibration of the 
horizontal flexible plate system. From this research, it was found that the ABC algorithm was modelled the system very well by achieving the lowest mean squared error, good correlation tests and high stability in the pole zero diagram as compared to RLS algorithm. However, the result of RLS modelling was proved to be biased and not correlated up to $95 \%$ confidence level. This correlation tests are very important in order to determine the effectiveness among the developed models. Here, it can be concluded that, artificial bee colony algorithm has successfully represented the approximate model of the horizontal flexible plate with all edges clamped boundary condition.

\section{Acknowledgement}

The authors would like to express their gratitude to Minister of Education Malaysia (MOE), Universiti Teknologi MARA (UiTM) and Universiti Teknologi Malaysia (UTM) for funding the research and providing facilities to conduct this research.

\section{References}

[1] Ismail R (2006), "Neuro modeling and vibration control of flexible rectangular plate structure", Master Thesis, Dept. of Applied mechanics, Faculty of mechanical Engineering, Universiti Teknologi Malaysia

[2] Zadeh MRS (2009), "Optimal placement of sensor and actuator for active vibration control of flexible structures", Master Thesis, Dept. of Applied mechanics, Faculty of mechanical Engineering, Universiti Teknologi Malaysia.

[3] Al-Khafaji AAM (2010), "System identification of flexible plate structure", Master Thesis, Dept. of Applied mechanics, Faculty of mechanical Engineering, Universiti Teknologi Malaysia.

[4] Mat Darus IZ \& Al-Khafaji AAM (2012), "Non-paramteric modeling of a rectangular flexible plate structure. Journal of Engineering Applications of Artificial Intelligence, 25: 94-106.

[5] Mat Darus IZ (2004), "Soft computing adaptive vibration control of flexible structures", Ph.D. Thesis, Department of Automatic Control and System Engineering, University of Sheffield.

[6] Hadi MS \& Mat Darus IZ (2013), "Intelligence swarm model optimization of flexible plate structure system", International Review of Automatic Control, 6(3): 322-331.

[7] Tavakolpour AR, Mat Darus IZ, Mailah M \& Tokhi MO (2010) "Genetic algorithm-based identification of transfer function parameters for a rectangular flexible plate system. Journal of Engineering Applications of Artificial Intelligence, 23(8): 1388-1397.

[8] Hadi MS, Hashim MH \& Mat Darus IZ (2012), "Genetic modeling of a rectangular flexible plate system with free-free-clampedclamped (FFCC) edges". IEEE Proceedings on Control, Systems and Industrial Informatics (ICCSII), Bandung, Indonesia, 23-26 September, pp. 173-179.

[9] Hadi, MS, Mat Darus IZ, Pek Eek RT \& Mohd Yatim H (2014), "Swarm intelligence for modeling a flexible plate structure system with clamped-clamped-free-free boundary condition edges". IEEE Symposium on Industrial Electronics \& Applications (ISIEA), 28 September-1 October, pp. 119-124 